02

\title{
Исследование индукционно-резонансного взаимодействия кластеров воды в минералах фторапатита методами поляризационной ИК и КР-спектроскопии
}

\section{(C) В.М. Золотарев}

Университет ИТМО, 197101 Санкт-Петербург, Россия

e-mail: VM-Zolotarev@mail.ru

Поступила в редакцию 18.04.2018 г.

\begin{abstract}
Методами инфракрасной (ИК) спектроскопии и спектроскопии комбинационного рассеяния (КР) проведены поляризационные исследования колебательных спектров кластеров $\left(\mathrm{H}_{2} \mathrm{O}\right)_{n}$ в образцах монокристаллического фторапатита разных месторождений. Измерения ИК и КР-спектров проведены для двух ортогональных направлений внешнего возбуждающего поля: параллельно и перпендикулярно $c$-каналам в монокристаллах фторапатита. Интерпретация взаимодействия внешнего электромагнитного поля с кластерами воды, находящимися в микропустотах и микротрещинах кристалла, со структурными группами F. ..HO апатита дана в рамках диполь-дипольного приближения. Показано, что это взаимодействие имеет индукционно-резонансный характер (метод ИК спектроскопии), обусловленный переносом колебательного возбуждения от групп F. . .HO к кластерам $\left(\mathrm{H}_{2} \mathrm{O}\right)_{n}$. Для метода КР-спектроскопии имеет место индукционный характер возбуждения кластеров $\left(\mathrm{H}_{2} \mathrm{O}\right)_{n}$ за счет передачи электронного возбуждения от групп $\mathrm{F}$...HO.
\end{abstract}

DOI: $10.21883 /$ OS.2018.08.46362.110-18

\section{Введение}

Исследованию воды в минералах, где вода не является структурным элементом, входящим в состав минерала, и присутствует только в малых количествах, уделяется большое внимание в силу той роли, которую ей приписывают в разных процессах генезиса Земли [1-3] и других планет Солнечной системы [4], а также космических объектов: спутников [5], астероидов [6] и метеоритов [7].

Одним из таких минералов является апатит - в нем молекулярная вода не входит в кристаллическую структуру, однако в кристалле может содержаться значительное количество нестехиометрической воды, которая наряду с другими примесями оказывает заметное влияние на свойства фторапатита. Вода в виде групп F. . .НО присутствует в $c$-каналах кристаллического фторапатита, а в молекулярной форме $\left(\mathrm{H}_{2} \mathrm{O}\right)_{n}$ - на границах роста кристаллитов, в субмикроскопических полостях и трещинах минерала апатита. Вода, присутствующая в апатите, который является основой костной ткани многих живых существ, играет важную роль, поддерживая стабильность механических свойств костной ткани при динамических нагрузках [8-11]. Поэтому изучение свойств разных типов молекул воды в апатите важно также для понимания регуляции ионного обмена и направленной диффузии небольших молекул вдоль сканалов, расположенных в кристаллах апатита, где находятся гидроксильные группы и соседствующие с ними кластеры воды [12,13].

Изучение взаимовлияния разных по свойствам типов воды, у которых сильно отличаются электрооптические параметры (дипольный момент, поляризуемость), существен для расчетов, связанных с количественной оценкой малых концентраций воды в рудах и минералах $[1,14]$. Особенно учет необходим в случаях, когда вода по стехиометрии не входит в состав минерала. Гидроксильные группы типа F...HО и О...НО имеют линейное строение и образуют комплексы, состоящие из несколько групп $\mathrm{F}$...HO(HO $)_{n}[15]$. Олигомеры нестехиометрической воды $\left(\mathrm{H}_{2} \mathrm{O}\right)_{n}$ образуют небольшие кластеры (димеры, тримеры, тетрамеры и др.), которые имеют замкнутое циклическое строение [16,17]. У групп типа F...НО и О...НО с их линейным строением значения производной $(\partial \mu / \partial Q)^{2}$ от дипольного момента $\mu$ по нормальной координате $Q$ (вдоль связи) и от поляризуемости $\alpha\left(\partial \alpha_{A} / \partial Q\right)$ отличаются в большую сторону от соответствующих параметров отдельных кластеров $\left(\mathrm{H}_{2} \mathrm{O}\right)_{n}$ с циклическим строением. В результате этих отличий интенсивность взаимодействия различных типов воды с внешним электромагнитным полем будет разной по величине. Однако, например, когда гидроксил и кластеры воды образуют одну систему, в результате диполь-дипольного взаимодействия у кластеров будет индуцироваться дополнительный дипольный момент. В итоге интенсивность взаимодействия кластеров воды с внешним полем усилится. Кроме того, если колебания гидроксила и кластеров воды будут иметь одну симметрию типа $A$, а частоты колебаний совпадают, то допускается резонансное взаимодействие между такого типа колебаниями [18-21]. Следует отметить, что механизм индуктивно-резонансных безызлучательных переходов между электронными уровнями широко используется для изучения люминесценции в конденсированных средах, содержащих редкоземельные элементы [22-25].

Основная цель настоящей работы состояла в изучении поляризационными методами механизма колебательно- 

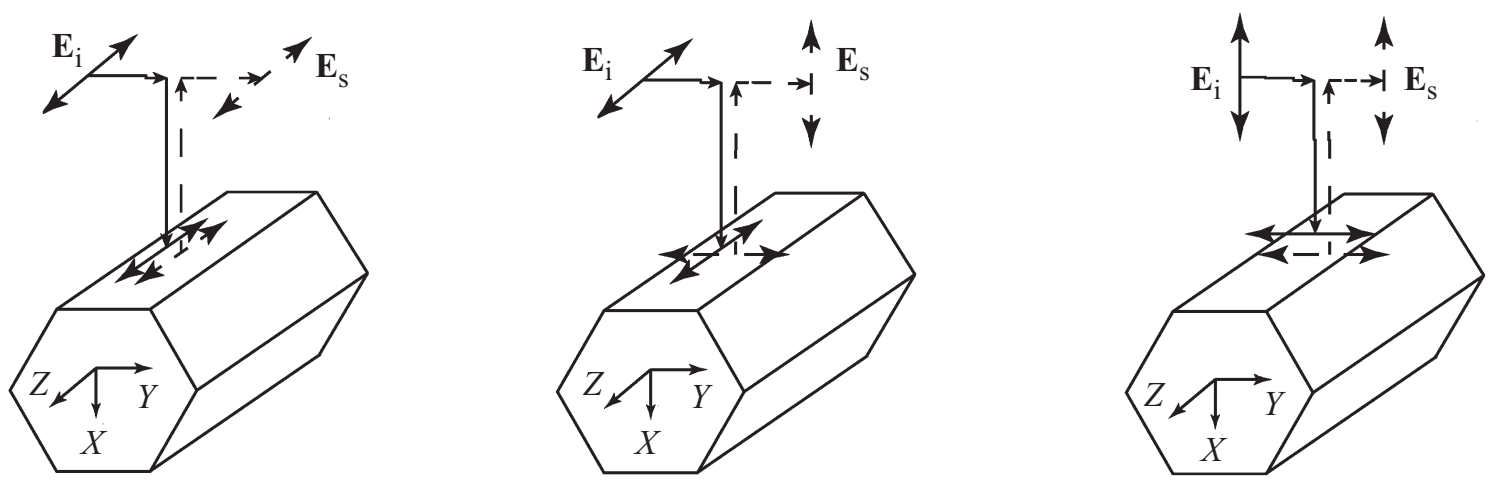

Рис. 1. Схема поляризационных измерений методом КР: $x(z z)-x$ (слева), $x(z y)-x$ (в центре), $x(y y)-x$ (справа). Вектор напряженности падающего линейно поляризованного излучения $\mathbf{E}_{i}$, вектор напряженности рассеянного поляризованного излучения $\mathbf{E}_{s}$.

го взаимодействия структурных групп типа F...HO, О...НО и соседствующими с этими группами нестехиометрических малых кластеров воды $\left(\mathrm{H}_{2} \mathrm{O}\right)_{n}$, присутствующих в микротрещинах монокристаллов апатита ряда месторождений.

\section{Образцы и аппаратура}

Характеристики монокристаллов фторапатита для экспериментальных исследований представлены в табл. 1. Схема поляризационных измерений спектров КР монокристаллов фторапатита дана на рис. 1. Спектры КР были зарегистрированы в геометрии рассеяния назад на приборе LabRam HR800 с дифракционной решеткой 1800 lines/mm и апертурой $20 \mu \mathrm{m}$. Для возбуждения КРС использовался Не-Nе-лазер $\left(\lambda_{\text {exc }}=632.8 \mathrm{~nm}\right)$. На образце под объективом $50 \times(N A=0.5)$ мощность лазерного излучения достигала $13 \mathrm{~mW}$. Для разделения фононных мод по симметрии осуществлялась съемка поляризованных спектров в геометриях $x(z z)-x, x(z y)-x$, $x(y y)-x$. Векторы напряженностей падающего $\left(\mathbf{E}_{i}\right)$ и рассеянного $\left(\mathbf{E}_{s}\right)$ излучения ориентированы по $c$ каналам с ОН-группами, положение которых совпадает с осью $Z$. Направление падающего и рассеянного света коллинеарно направлению оси $X$ (рис. 1$)$.

Использование в эксперименте монокристаллов позволяло проводить поляризационные измерения ИК и КР-спектров и анализировать влияние анизотропии фторапатита в области поглощения структурной воды на взаимодействие внешнего поля Е с кластерами воды и группами типа F. . НО, О. . .НО, ориентированных вдоль $c$-каналов. Обоснование наличия в кристаллах фторапатита структурно неупорядоченных маленьких кластеров воды с разной концентрацией базировалось на сопоставлении результатов измерений ИК и КР-спектров образцов фторапатита и кристаллического кварца, полученных из разных месторождений и разными методами (рис. 2,a). В работе [2] методом ИК спектроскопии с помощью дейтерирования показано, что нестехиометрическая вода в кристаллическом кварце присутствует пре- имущественно в открытых трещинах и полостях. Присутствие в спектрах кварца [3,26] и апатита [27] одинаковых частот в области $4000-3000 \mathrm{~cm}^{-1}$ позволяет сделать вывод о единой природе нестехиометрической воды в этих минералах. Существенно, что в ИК и КР-спектрах образцов (табл. 1) при смене поляризации внешнего излучения положение частот оставалось неизменным. Различие состояло преимущественно в интенсивности полос, что связано с разной концентрацией отдельных структурных элементов, образующих кластеры воды в кварце и фторапатите. Дополнительным подтверждением сказанного может служить совпадение частот в ИК и КР-спектрах в диапазоне $4000-3000 \mathrm{~cm}^{-1}$ для образцов апатита разных месторождений (табл. 1), полученных независимо разными методами: поглощения, диффузного и зеркального отражения от плоской пластинки [27-29] (рис. 2, b). Спектр диффузного отражения [29] для удобства сопоставления преобразован с помощью функции Кубелки-Мунка [30]. Данные о ходе поглощения вычислялись из спектра зеркального отражения [28] плоской пластинки. Величина спектральной абсорбции слабо поглощающей пластинки толщиной $d$ при условии $n \gg k$ в соответствии с выражением $A=1-T-R(T-$ пропускание, $R$ - отражение пластинки) может быть получена [31] из формулы

$$
A=\frac{(1-R)[1-\exp (-K d)]}{1-R^{2} \exp (-2 K d)},
$$

где $K-$ бугеровский коэффициент поглощения, определяемый из соотношения $K=\frac{4 \pi k}{\lambda}, k-$ мнимая часть комплексного показателя преломления.

Из сопоставления кривых, приведенных на рис. 2, $b$ и полученных тремя разными методами для разных образцов монокристаллического апатита, видна близость частот основных полос для сопоставляемых образцов. Кроме того, как видно из рис. 2, $b$, имеется хорошее сходство по частотам также и для порошка нанокристаллического гидроксилапатита [32]. Сходство ИК спектров указывает на общую природу полос воды для сопоставляемых минералов в области $4000-3000 \mathrm{~cm}^{-1}$. 

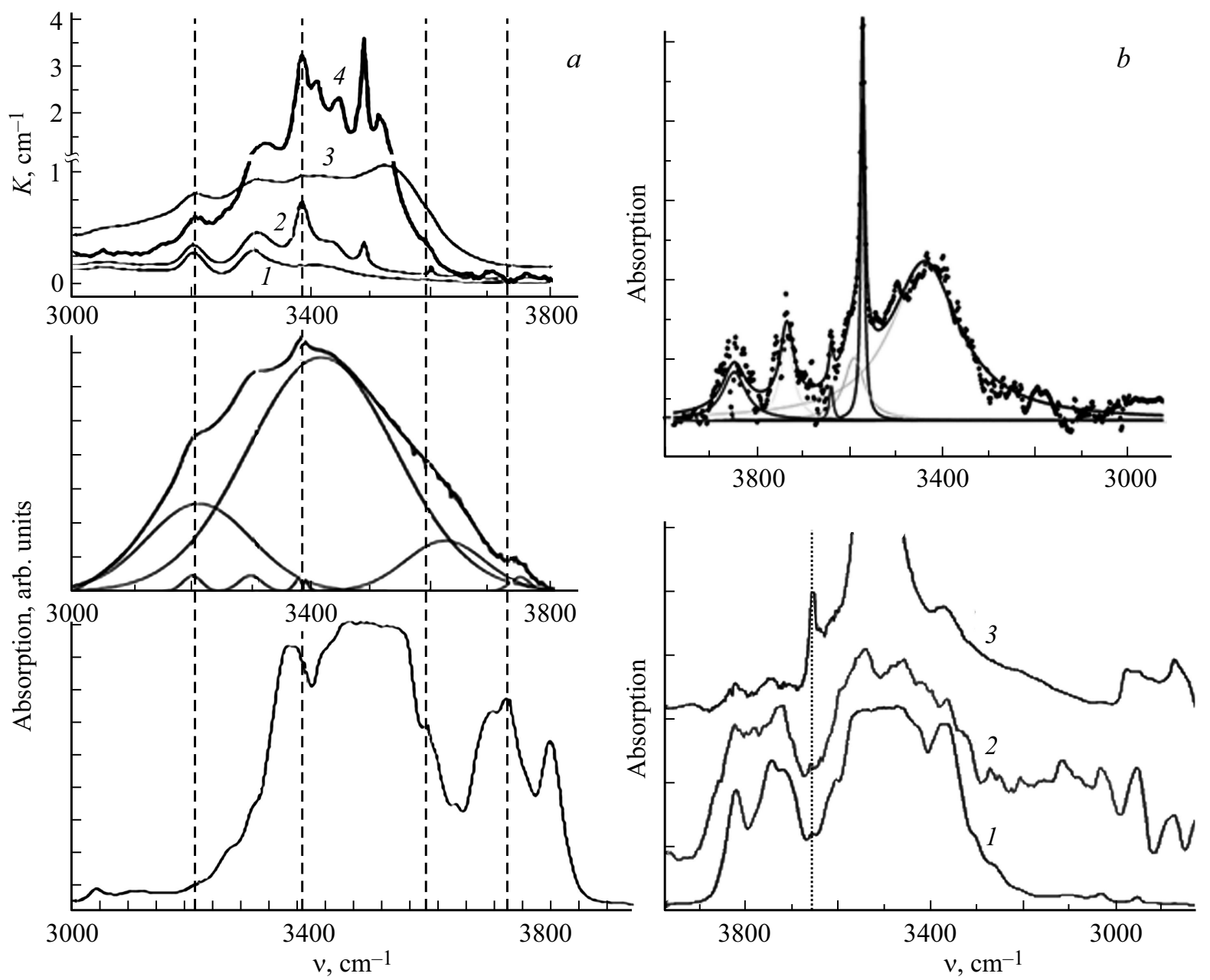

Рис. 2. (a) Инфракрасные спектры поглощения в области $4000-2800 \mathrm{~cm}^{-1}$ : (вверху) различные $(1-4)$ монокристаллы кварца [26]; (в центре) Кузнечихинское месторождение кварца, Урал, моделирование ИК спектра выполнено суперпозицией гауссовых линий [3]; (внизу) Хибины, Кольский полуостров, образец 1S (табл. 1) [27]. (b) Инфракрасный спектр поглощения порошка гидроксилапатита [32] (вверху); ИК спектры фторапатита (табл. 1) в области 4000-2800 $\mathrm{cm}^{-1}$, полученные методами: 1 поглощение, образец $1 \mathrm{~S}$ (толщина $\sim 2 \mathrm{~mm}$ ), $\mathbf{E} \| c$ [27]; 2 - диффузное отражение (спектр обработан по формуле Кубелки-Мунка), образец 6S (порошок, фракция 20-125 $\mu \mathrm{m}$ [29]; 3 - зеркальное отражение, образец 5S, не ориентирован (тонкая пластинка) [28].

Таблица 1. Характеристики монокристаллов фторапатита

\begin{tabular}{|c|c|c|c|c|}
\hline № & Название & Химический состав & Габитус и цвет & Происхождение \\
\hline $1 \mathrm{~S}$ & Фторапатит & $\mathrm{Ca}_{5.00}\left(\mathrm{P}_{1.00} \mathrm{O}_{4}\right)_{3}\left(\mathrm{~F}_{0.98} \mathrm{OH}_{0.01} \mathrm{Cl}_{0.01}\right)$ & $\begin{array}{c}\text { Призматический, } \\
\text { прозрачный }\end{array}$ & $\begin{array}{c}\text { Россия, Хибины, } \\
\text { Кольский полуостров }\end{array}$ \\
\hline $2 \mathrm{~S}$ & Фторапатит & $\begin{array}{c}\left(\mathrm{Ca}_{4.4} \mathrm{Sm}_{0.30} \mathrm{Mn}_{0.10} \mathrm{Na}_{0.15} \mathrm{Cs}_{0.05}\right)_{\Sigma=5} \times \\
\left(\left(\mathrm{P}_{0.96} \mathrm{Si}_{0.04}\right)_{\Sigma=1}\right)_{3} \mathrm{~F}_{1.00}\end{array}$ & $\begin{array}{l}\text { Призматический, } \\
\text { сине-зеленый, } \\
\text { непрозрачный }\end{array}$ & $\begin{array}{c}\text { Россия, Хибины, } \\
\text { Кольский полуостров }\end{array}$ \\
\hline $3 \mathrm{~S}$ & $\begin{array}{c}\text { Карбонат } \\
\text { фторапатит }\end{array}$ & $\mathrm{Ca}_{5}\left(\mathrm{PO}_{4}, \mathrm{CO}_{3}\right)_{3} \mathrm{~F}$ & $\begin{array}{c}\text { Призматический, } \\
\text { темно-серый } \\
\text { полупрозрачный }\end{array}$ & $\begin{array}{l}\text { Россия, Прибайкалье, } \\
\text { Слюдянка }\end{array}$ \\
\hline $4 \mathrm{~S}$ & Фторапатит & $\mathrm{Ca}_{5.00}\left(\mathrm{P}_{1.00} \mathrm{O}_{4}, \mathrm{CO}_{3}\right)_{3}\left(\mathrm{~F}_{0.90} \mathrm{OH}_{0.1}\right)$ & $\begin{array}{c}\text { Таблитчатый, } \\
\text { облитерированный, } \\
\text { прозрачный }\end{array}$ & $\begin{array}{l}\text { Россия, Апатиты, } \\
\text { Кольский плуостров }\end{array}$ \\
\hline $5 \mathrm{~S}$ & Фторапатит & $\mathrm{Ca}_{5.00}\left(\left(\mathrm{P}_{0.98} \mathrm{Si}_{0.01} \mathrm{~S}_{0.01}\right) \mathrm{O}_{4}\right)_{3} \mathrm{~F}_{1.00}$ & $\begin{array}{l}\text { Pale yellow short } \\
\text { hexagonal prism }\end{array}$ & $\begin{array}{c}\text { Cerro de Mercado mine, } \\
\text { Durango Mexico }[28]\end{array}$ \\
\hline $6 \mathrm{~S}$ & Фторапатит & $\mathrm{Ca}_{5}\left(\mathrm{PO}_{4}\right)_{3} \mathrm{~F}$ & $\begin{array}{l}\text { Particle Size } \\
20-125 \mu \mathrm{m}\end{array}$ & $\begin{array}{c}\text { Canada, Ontario, Lanark } \\
\text { County, Perth [29] }\end{array}$ \\
\hline
\end{tabular}




\section{Результаты эксперимента}

\section{Данные ИК спектроскопии}

При получении ИК спектра монокристалл апатита ориентировался относительно ортогональных осей $X Y Z$. Направление падающего пучка соответствовало оси $X$, вектор $\mathbf{E}$ поляризованного излучения поочередно ориентировался вдоль оси $Z$, а затем вдоль оси $Y$. Инфракрасные спектры пропускания образцов апатитов в диапазоне $5000-350 \mathrm{~cm}^{-1}$ были получены на ИК фурье-спектрометре TENSOR 27 фирмы Bruker с разрешением $2 \mathrm{~cm}^{-1}$ для диафрагмы $2 \mathrm{~mm}$ при 500 сканах в поляризованном излучении. Использовались дифракционные поляризаторы 1200 и 600 lines $/ \mathrm{mm}$ на подложках $\mathrm{CaF}_{2}$ и пленке из полиэтилена. Особенность спектров, полученных для двух образцов $1 \mathrm{~S}$ и $2 \mathrm{~S}$ (табл. 1), состояла в сильном дихроизме. Эта зависимость для образца $1 \mathrm{~S}$ отображена в диапазоне $4000-3000 \mathrm{~cm}^{-1}$ на рис. 3. В обоих случаях поглощение излучения при $\mathbf{E} \| Z(Z \| c)$ значительно превышало поглощение при $\mathbf{E} \perp Z$, однако форма контура сложной полосы в диапазоне $4000-3000 \mathrm{~cm}^{-1}$ практически оставалась неизменной. Причем полоса $3539 \mathrm{~cm}^{-1}$, относящаяся к группе F...HО, имеет наи-
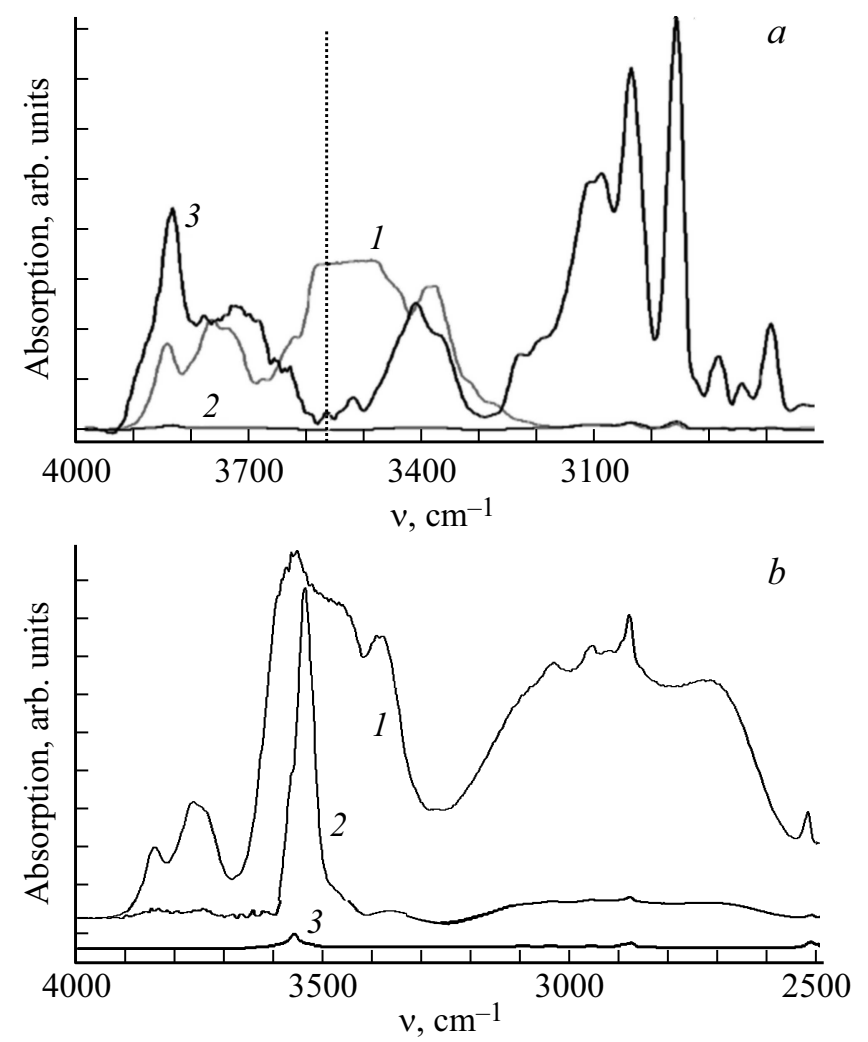

Рис. 3. (a) Инфракрасные спектры поглощения фторапатита, образец $1 \mathrm{~S}$, в области $4000-2800 \mathrm{~cm}^{-1}: 1-\mathbf{E} \| c$, толщина образца $\sim 2 \mathrm{~mm} ; 2-\mathbf{E} \perp c ; 3-\mathbf{E} \| c, \times 50$ масштабирование шкалы ординат. В области $3100-2800 \mathrm{~cm}^{-1}$ видны сильные полосы углеводородов. $(b)$ Инфракрасные спектры поглощения фторапатита, образец 4S, в области $4000-2500 \mathrm{~cm}^{-1}: 1-$ $\mathbf{E} \| c$, толщина образца $2 \mathrm{~mm} ; 2-\mathbf{E} \| c$, толщина образца $20 \mu \mathrm{m} ; 3$ - толщина образца $2 \mathrm{~mm}, \mathbf{E} \perp c$.

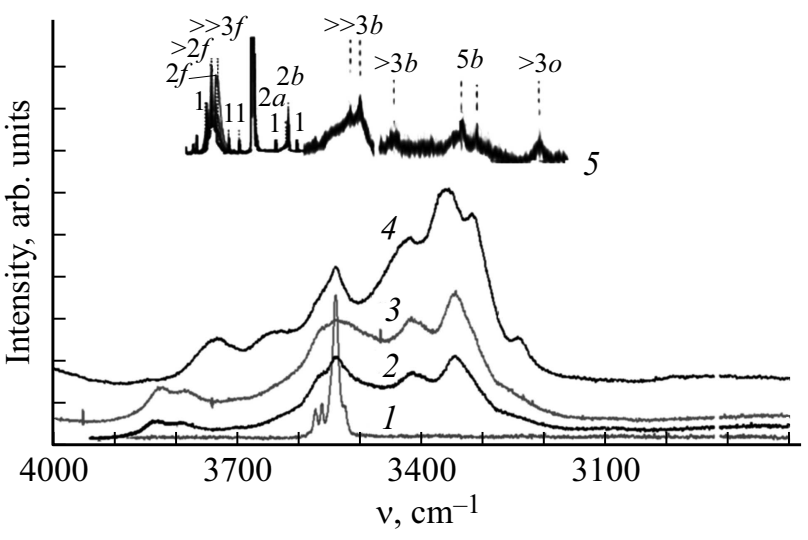

Рис. 4. Спектры КР монокристаллов фторапатита $1 \mathrm{~S}$ (1), $3 \mathrm{~S}(2), 4 \mathrm{~S}(3), 2 \mathrm{~S}(4)$ в области $4000-2800 \mathrm{~cm}^{-1}$ для конфигурации поляризатор-анализатор $x[z z]-x$, толщина образца $2 \mathrm{~mm}, \mathbf{E} \perp c ; 5-$ спектр кластеров $\left(\mathrm{H}_{2} \mathrm{O}\right)_{n}(n=2-5)$, полученных охлаждением паров воды в смеси с благородными газами $\mathrm{He}, \mathrm{Ne} / \mathrm{He}, \mathrm{Ar} / \mathrm{He}[33]$, числа над кривой означают размерность кластера $n$, буквенные обозначения полос: $b-$ связь ОН. . .,$f-$ свободная группа $\mathrm{OH}, a-$ акцептор, $o-$ деформационный обертон.

больший дихроизм, что хорошо видно при растяжке спектра, полученного при $\mathbf{E} \perp Z$ (рис. 3, a). Сильное различие в интенсивностях полос для спектров в положениях $\mathbf{E} \| Z$ и $\mathbf{E} \perp Z$ свидетельствует о сильном влиянии группы F...НО, ориентированной параллельно оси $c$ кристалла, на поглощательную способность кластеров воды $\left(\mathrm{H}_{2} \mathrm{O}\right)_{n}$, ассоциированных с этой группой. Поскольку ИК поглощение пропорционально $(\partial \mu / \partial Q)^{2}$, то судя по интенсивности ИК спектров, этот параметр намного больше для спектра с ориентацией $\mathbf{E} \| Z$ по сравнению с ориентацией $\mathbf{E} \perp Z$. Кроме того, сходство сопоставляемых поляризованных ИК спектров монокристалла и не ориентированных частиц порошка гидроксилапатита [32] подтверждает ранее сделанный вывод, основанный на независимости набора частот от направления вектора $\mathbf{E}$ внешнего излучения, что кластеры воды $\left(\mathrm{H}_{2} \mathrm{O}\right)_{n}$ в образце $1 \mathrm{~S}$ не имеют преимущественной ориентации в порах и трещинах, т. е. распределены изотропно.

Наличие дефектов и примесей в кристалле апатита приводит к образованию связей типа Ме..ОН...F , где $\mathrm{Me}=\mathrm{Li}, \mathrm{K}, \mathrm{Na}, \mathrm{Mn}$ и др., что вызывает отклонение линейной группы F. ..НО от исходного положения, совпадающего с направлением оси $c$. В результате дихроизм полосы $3539 \mathrm{~cm}^{-1}$ становится заметно меньше, что характерно для ИК спектра образца $2 \mathrm{~S}$, в котором присутствует много примесей (рис. $3, b)$.

\section{Данные КР-спектроскопии}

Спектры КР в диапазоне $4000-2800 \mathrm{~cm}^{-1}$ были получены с использованием поляризованного излучения в рамках схемы рис. 1 для четырех монокристаллов фторапатита $1 \mathrm{~S}-4 \mathrm{~S}$ (табл. 1). Усредненные по разным точкам образца данные для частот образцов $1 \mathrm{~S}-4 \mathrm{~S}$ представлены на рис. 4 и в табл. 2, где проведена интерпретация 
Таблица 2. Сопоставление основных полос в ИК и КР-спектрах $\left(\mathrm{H}_{2} \mathrm{O}\right)_{n}$ и $\mathrm{H}^{+}\left(\mathrm{H}_{2} \mathrm{O}\right)_{n}$ в монокристаллах (образцы $\left.1 \mathrm{~S}-4 \mathrm{~S}\right)$ с данными эксперимента, полученными для кластеров воды $\left(\mathrm{H}_{2} \mathrm{O}\right)_{n}, n=2-5$ методом низкотемпературной матричной изоляции

\begin{tabular}{|c|c|c|c|c|c|}
\hline \multirow{2}{*}{$\begin{array}{c}\text { Наши данные (КР) } \\
v, \mathrm{~cm}^{-1}\end{array}$} & \multicolumn{2}{|c|}{ КР [33] } & \multirow{2}{*}{$\frac{\text { ИК [34] }}{v, \mathrm{~cm}^{-1}}$} & \multirow{2}{*}{$\frac{\text { ИК [32] }}{2, \mathrm{~cm}^{-1}}$} & \multirow{2}{*}{ ИК [12] } \\
\hline & $v, \mathrm{~cm}^{-1}$ & отнесение полос & & & \\
\hline 3924 осл & & $1 \mathrm{f}$ & & $3642,3737,3866$ & \\
\hline $3845-3825 \mathrm{cp}$ & & & $2 n, 3 n$ & & $3925-3901$ \\
\hline & & & $2 n$ & & $3857-3802$ \\
\hline $3780 \mathrm{cp}$ & & & $2 n$ & & 3754 \\
\hline 3735 & 3730 & $2 \mathrm{f}$ & $2 n$ & & 3731 \\
\hline & 3721 & $>2 \mathrm{f}$ & $3 n$ & & \\
\hline & 3711 & $\gg 3 \mathrm{f}$ & & & \\
\hline & 3657 & 1 & & & \\
\hline $3634 \mathrm{cp}$ & 3651 & $2 \mathrm{a}$ & & & 3673 \\
\hline 3616 пл & 3606 & $2 b^{*}$ & $4 n$ & & \\
\hline $3570 \mathrm{cp}$ & & & & & \\
\hline & 3602 & $2 b$ & & $3526-3598$ & \\
\hline $3562 \mathrm{c}$ & & & & & \\
\hline & 3548 & $3 b(I R-w)$ & & & \\
\hline $3540 \mathrm{oc}$ & 3533 & $3 \mathrm{~h}(\mathrm{IR})$ & & & \\
\hline $3526 \mathrm{cp}$ & 3533 & $3 b(1 R)$ & & & \\
\hline & 3506 & $\gg 3 b$ & $5 n$ & & \\
\hline & 3491 & $3 b$ & & & \\
\hline & 3438 & $>3 b(4 b ?)$ & & & $3442-3432$ \\
\hline $3416 \mathrm{c}$ & 3401 & $4 \mathrm{~b}(\mathrm{IR})$ & & $3420-3450$ & \\
\hline & - & $>3 b(5 b ?)$ & & & \\
\hline $3360-3346$ oc & 3355 & $5 \mathrm{~b}(\mathrm{IR})$ & & & \\
\hline & 3347 & $4 b^{*}$ & & & \\
\hline $3316 \mathrm{c}$ & 3334 & $4 b$ & & & 3323 \\
\hline & 3310 & $5 b$ & & $3237-3263$ & \\
\hline $3244 \mathrm{cp}$ & 3214 & $>30$ & & & 3231 \\
\hline 3158 сл & 3153 & 10 & & & \\
\hline & 3121 & $\gg 3 b$ & & & \\
\hline 3076 сл & & & & & \\
\hline 2940-2840 диф & & & $\mathrm{H}^{+}\left(\mathrm{H}_{2} \mathrm{O}\right)_{n=5}$ & 2940 & \\
\hline $2660 \mathrm{cp}$ & & & $\mathrm{H}^{+}\left(\mathrm{H}_{2} \mathrm{O}\right)_{n=4}$ & & \\
\hline $2386 \mathrm{cp}$ & & & $\mathrm{H}^{+}\left(\mathrm{H}_{2} \mathrm{O}\right)_{n=3}$ & & \\
\hline
\end{tabular}

Примечание. Обозначения: b — для связи ОН. . .О, f - для свободной группы ОН, а — для акцептора, о - для деформационного обертона, * „горячие“ переходы, (IR) —- активность колебаний в ИК спектре. Обозначения интенсивностей полос: ос - очень сильная, с - сильная, ср — средняя, сл - слабая, осл - очень слабая, пл - плечо, диф - диффузная.

полос в рамках кластерной схемы строения воды $\left(\mathrm{H}_{2} \mathrm{O}\right)_{n}$ в соответствии с литературными данными [33,34].

Особенность спектров КР в диапазоне $4000-3000 \mathrm{~cm}^{-1}$ заключалась в значительном различии интенсивностей $(I)$ полос для компонент излучения, рассеянного в ортогональных направлениях $\left(I_{z z}\right.$ и $\left.I_{z y}\right)$ и $\left(I_{y y}\right.$ и $\left.I_{y z}\right)$ соответственно вдоль и поперек $c$-каналов образца (рис. 5). В первом положении $\left(I_{z z}\right.$ и $\left.I_{z y}\right)$ ось поляризатора параллельна оси $Z$ (анализатор соответственно направлен по осям $Z$ и $Y)$. Во втором положении $\left(I_{y y}\right.$ и $I_{y z}$ ) ось поляризатора параллельна $Y$ (анализатор соответственно направлен по осям $Y$ и $Z$ ) (рис. 1). Так же как и в ИК спектрах, наиболее интенсивные полосы в спектрах КР для всех образцов наблюдались при $\mathbf{E} \| Z$, т.е. для компоненты рассеянного излучения $I_{z z}$. Наибольшие изменения в форме спектра наблюдались в окрестности полосы $3539 \mathrm{~cm}^{-1}$. Интенсивности $I_{z y}$ и $I_{y z}$ этой полосы были всегда меньше, чем $I_{z z}$ и $I_{y y}$ (рис. 5). Поскольку $I \sim(\partial \alpha / \partial Q)^{2}$, то на основании поляризованных спектров КР можно заключить, что для элементов тензора поляризуемости колебаний, которым отвечает полоса $3539 \mathrm{~cm}^{-1}$, выполняется следующее соотношение:

$$
\left|\alpha_{z y}\right|<\left|\alpha_{y y}\right|<\left|\alpha_{z z}\right|
$$

Степень деполяризации $\rho=I_{\perp} / I_{\|}$рассеянного излучения для рассмотренных двух положений поляризатор-анализатор $\left(0^{\circ}\right.$ и $\left.90^{\circ}\right)$ относительно направления $c$-каналов образца определяется соответственно из соотношений

$$
\rho=I_{z y} / I_{z z}, \quad \rho=I_{y z} / I_{y y} .
$$



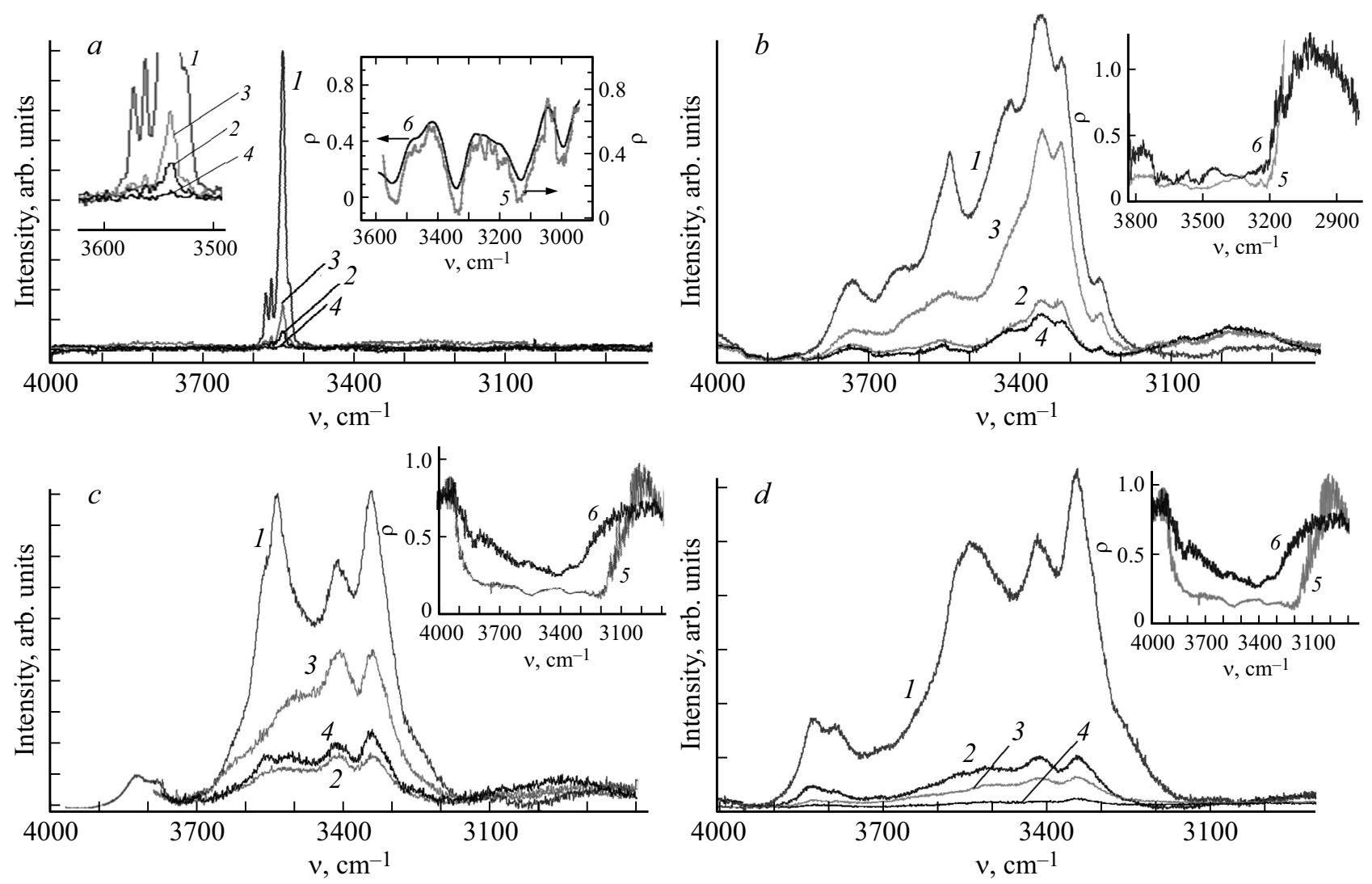

Рис. 5. Спектры КР (1-4) и степень поляризации $\rho(5,6)$ монокристаллов фторапатита $(a) 1 \mathrm{~S}$ (слева на вставке для наглядности дан спектр с масштабированием обеих шкал), (b) $3 \mathrm{~S},(c) 4 \mathrm{~S},(d) 2 \mathrm{~S}$ в области 4000-2800 $\mathrm{cm}^{-1}$ для конфигураций поляризаторанализатор $x[z z]-x(1), x[z y]-x(2), x[y y]-x(3), x[y z]-x(4) ; \rho=\frac{I\{x[z y]-x\}}{I\{x[z z]-x\}}(5), \rho=\frac{I\{x[y z]-x\}}{I\{x[y y]-x\}}(6)$.

Степени деполяризации $\rho$ образцов $1 \mathrm{~S}-4 \mathrm{~S}$ представлены на рис. 5. Из теории [18-21] следует, что если падающее излучение линейно поляризовано, то для $\rho \leq 3 / 4$ соответствующая линия в спектре считается „поляризованной“ и данное колебание является симметричным. Если $\rho \geq 3 / 4$, соответствующая линия в спектре считается „неполяризованной“ и колебание является несимметричным. Из рис. 5 видно, что в центральной части диапазона $4000-3000 \mathrm{~cm}^{-1}$ параметр $\rho$ для геометрии $I_{z y} / I_{z z}$ меньше, чем для геометрии $I_{y z} / I_{y y}$. Это показывает, что колебания $v_{1}$ группы F...HO, имеющие $A$ симметрию, более активно возбуждают симметричные колебания кластеров $\left(\mathrm{H}_{2} \mathrm{O}\right)_{n}$. Примечательно, что параметр $\rho$ для полосы $3539 \mathrm{~cm}^{-1}$, соответствующей группе F...HO, для всех четырех образцов апатита меньше, чем параметр $\rho$ для наиболее интенсивной полосы $3350 \mathrm{~cm}^{-1}$ кластерной воды (табл. 3). В табл. 3 для сопоставления приведены также величины $\rho$ для жидкой воды $[35,36]$.

При рассмотрении табл. 3 видно, что для полосы $3539 \mathrm{~cm}^{-1}$ наблюдается корреляция между величиной $\rho$ и полушириной $\Delta v$ этой полосы: увеличение $\Delta v$ приводит к росту $\rho$. Это указывает, что примеси и дефекты кристаллической решетки апатита приводят к понижению $A$-симметрии колебания $v_{1}$ группы F. . НО.
Для диапазонов $4000-3750$ и $3100-2800 \mathrm{~cm}^{-1}$ значения $\rho>3 / 4$, что указывает на $E$-симметрию колебаний в этих областях. Полосы в области $4000-3750 \mathrm{~cm}^{-1}$ относятся к антисимметричным колебаниям свободных гидроксилов [34,35]. Диффузная полоса $2960-2900 \mathrm{~cm}^{-1}$ шириной $\approx 300 \mathrm{~cm}^{-1}$ относится к комплексу гидроксония типа $\mathrm{H}_{3} \mathrm{O}^{+}\left(\mathrm{H}_{2} \mathrm{O}\right)_{n}$ [37-40]. Интенсивность этой полосы наибольшая для $I_{y z}$ (вектор напряженности возбуждающего излучения $\mathbf{E} \perp Z$ ), что связано с ориентацией этого комплекса относительно $c$-каналов апатита.

\section{Обсуждение результатов}

Изменения интенсивностей полос ИК и КР-спектров в области валентных колебаний воды в кристаллическом апатите можно объяснить исходя из схемы взаимодействия системы двух разных типов осцилляторов (диполей) D (донор) и А (акцептор) с высокочастотным внешним электрическим полем E [18-21]. Осцилляторы D и А характеризуются системой перекрывающихся основных колебательных уровней. Осциллятор D имеет относительно узкую ширину основного уровня, а осциллятор А имеет систему широких перекрывающихся уровней. Электрооптические свойства осцилляторов D 
Таблица 3. Степень деполяризации $\rho$ в КР-спектре полос 3539 (группа F...НО) и $3350 \mathrm{~cm}^{-1}$ (пентамер воды) для минералов фторапатита и жидкой воды

\begin{tabular}{|c|c|c|c|c|c|c|c|c|c|c|c|c|}
\hline Образцы & \multicolumn{2}{|c|}{$1 \mathrm{~S}$} & \multicolumn{2}{|c|}{$2 S$} & \multicolumn{2}{|c|}{$3 \mathrm{~S}$} & \multicolumn{2}{|c|}{$4 \mathrm{~S}$} & \multicolumn{2}{|c|}{ вода [36] } & \multicolumn{2}{|c|}{ вода [35] } \\
\hline Полосы, $\mathrm{cm}^{-1}$ & 3539 & 3350 & 3539 & 3350 & 3539 & 3350 & 3539 & 3350 & 3500 & 3200 & 3410 & 3258 \\
\hline $\mathbf{E} \| Z$ & $0.07 \pm 0.02$ & $0.08 \pm 0.02$ & 0.08 & 0.16 & 0.11 & 0.15 & 0.14 & 0.16 & & & & \\
\hline $\mathbf{E} \perp Z$ & $0.13 \pm 0.05$ & $0.24 \pm 0.05$ & 0.15 & 0.20 & 0.66 & 0.47 & 0.46 & 0.43 & & & & \\
\hline$\Delta v, \mathrm{~cm}^{-1}$ & 13 & 47 & 47 & $50-80$ & $25-35$ & 49 & $\approx 70$ & 96 & 0.4 & 0.13 & 0.14 & 0.09 \\
\hline
\end{tabular}

Примечание. Полуширины $\Delta v$ полос 3539 и $3350 \mathrm{~cm}^{-1}$ были получены путем разложения результирующего контура КР-спектров на составляющие с помощью программы OriginPro 8.

и А заметно различаются: $\mu_{\mathrm{D}} \gg \mu_{\mathrm{A}}, \partial \mu_{\mathrm{D}} / \partial Q>\partial \mu_{\mathrm{A}} / \partial Q$, $\alpha_{\mathrm{D}} \gg \alpha_{\mathrm{A}}, \partial \alpha_{\mathrm{D}} / \partial Q>\partial \alpha_{\mathrm{A}} / \partial Q$. Осцилляторы $\mathrm{D}$ (группа F...НО) фиксированы внутри $c$-каналов, их направление совпадает с осью $Z$, которая параллельна $c$-каналам в кристалле апатита. Осцилляторы А (кластеры $\mathrm{H}_{2} \mathrm{O}_{n}$ циклического типа) хаотически распределены в виде ассоциатов (включающих осцилляторы D), которые находятся в небольших порах с размерами $1-5 \mathrm{~nm}$. Динамика взаимодействия системы, состоящей из осцилляторов $\mathrm{D}$ и $\mathrm{A}$, с внешним полем $\mathbf{E}$ сопровождается передачей колебательного (ИК спектроскопия) и электронноколебательного (КР-спектроскопия) возбуждения от осцилляторов типа $\mathrm{D}$, более активно взаимодействующих с полем, к осцилляторам А, а от них далее к периферийным осцилляторам всего кластера. Такое дипольдипольное взаимодействие осцилляторов $\mathrm{D}$ и А будет сопровождаться посредством индукционного механизма дополнительной поляризацией диполей типа А в силу различия в поляризуемости $\left(\alpha_{\mathrm{D}}>\alpha_{\mathrm{A}}\right)$ соседствующих диполей. Следствием этого у диполей типа А возрастут дипольные моменты $\mu_{\mathrm{A}}$ и поляризуемости $\alpha_{\mathrm{A}}$, а вместе с ними увеличатся производные $\partial \mu_{\mathrm{A}} / \partial Q$ и $\partial \mu_{\mathrm{D}} / \partial Q$, что приведет к усилению взаимодействия диполей А с внешним полем $\mathbf{E}$. Выключение диполя D из взаимодействия с диполями А будет приводить к ослаблению взаимодействия поля с диполями А, в итоге интенсивность полос диполей А в ИК и КР-спектрах будет уменьшаться. В методе ИК спектроскопии полное „выключение“ диполя $\mathrm{D}$ из взаимодействия с диполями А возможно путем поворота поляризатора на $90^{\circ}$ от исходного положения (E направлено параллельно $c$-каналам).

Для метода КР-спектроскопии „выключение“ диполя $\mathrm{D}$ возможно только частично, поскольку три главные составляющие $\alpha_{x x}, \alpha_{y y}, \alpha_{z z}$ матрицы поляризуемости $\alpha_{\mathrm{D}}$ не равны 0. Однако, поскольку для группы F...НО в силу осевой симметрии $\alpha_{z z} \gg \alpha_{x x} \approx \alpha_{y y}$, изменение направления вектора $\mathbf{E}$ возбуждающего лазерного излучения от $Z$ на $90^{\circ}$ дает, например, для образца $3 \mathrm{~S}$ изменение регистрируемого сигнала до 7 раз (рис. 5). Существенно отметить, что интенсивности $I_{z z}$ для основных полос 3539 и $3350 \mathrm{~cm}^{-1}$ в спектрах образцов $2 \mathrm{~S}-4 \mathrm{~S}$ близки (рис. 5), т.е. происходит выравнивание этих компонент в результате индукционного взаимо- действия диполей D и А. Напротив, для $I_{y y}$, как и следовало ожидать исходя из схемы индукционного взаимодействия диполей $\mathrm{D}$ и $\mathrm{A}$, такой эффект в тех же спектрах отсутствует.

Для метода ИК спектроскопии усиление сигнала при повороте поляризатора от направления оси $Z$ на $90^{\circ}$ превышает 50 раз, что заметно больше, чем для метода КР-спектроскопии. Это усиление взаимодействия группы $\mathrm{F} . . \mathrm{HO}$ и кластеров $\left(\mathrm{H}_{2} \mathrm{O}\right)_{n}$ связано в рамках метода ИК спектроскопии с дополнительным влиянием резонанса Ферми, поскольку для этого выполняются необходимые условия: совпадение частот взаимодействующих молекулярных группировок, одинаковая симметрия колебаний и ассоциация группировок [18-21].

Независимым подтверждением рассмотренной схемы индукционного взаимодействия диполей $\mathrm{D}$ и А могут служить данные, касающиеся степени деполяризации спектров КР. Из табл. 3 можно заметить, что для всех исследованных образцов величина $\rho$ наименьшая при $\mathbf{E} \| Z$, что соответствует параллельности вектоpa $\mathbf{E}$ направлению наибольшего изменения величины $\partial \alpha_{\mathrm{D}} / \partial Q\left(\alpha_{\mathrm{D}}=\alpha_{z z}\right)$. В этом случае в соответствии с минимальным значением величины $\rho$ возбуждаются преимущественно симметричные колебания кластеров $\left(\mathrm{H}_{2} \mathrm{O}\right)_{n}$. При смене направления напряженности поля на $\mathbf{E} \perp Z$, т. е. при параллельности вектора $\mathbf{E}$ направлению изменения величины $\delta \alpha_{\mathrm{A}} / \delta Q\left(\alpha_{\mathrm{A}}=\alpha_{y y}\right), \rho$ увеличивается, что соответствует преимущественно возбуждению антисимметричных колебаний кластеров $\left(\mathrm{H}_{2} \mathrm{O}\right)_{n}$. Причина этих особенностей возбуждения разных колебаний связана с тем, что при $\mathbf{E} \| Z$ индукционно усиливаются симметричные колебания кластеров $\left(\mathrm{H}_{2} \mathrm{O}\right)_{n}$, поскольку для этого направления $\mathbf{E}$ колебания группы F...HO (диполь D) также симметричны (имеют А-симметрию). Для ориентации поля $\mathbf{E} \perp Z$ колебания группы F...HO носят смешанный характер, т.е. возбуждаются $A$ и $\mathbf{E}$ колебательные моды этой группы, что в свою очередь индуцирует как симметричные, так и антисимметричные колебания кластеров $\left(\mathrm{H}_{2} \mathrm{O}\right)_{n}$. Следует отметить, что для двух ориентаций $\mathbf{E} \| Z$ и $\mathbf{E} \perp Z$ в области $4000-3100 \mathrm{~cm}^{-1}$ значения $\rho<3 / 4$. Для этих ориентаций $\mathbf{E}$ относительно оси $Z$ в центре полос 3539 и $3350 \mathrm{~cm}^{-1}$ значения $\rho$ достигали минимумов $(\mathbf{E} \| Z)$ и 
максимумов $(\mathbf{E} \perp Z)$ на фоне общего глобального минимума кривой $\rho$. Причина такого поведения $\rho$ связана с большим количеством (более 9, рис. 5) перекрывающихся колебательных мод кластеров $\left(\mathrm{H}_{2} \mathrm{O}\right)_{n}$, а также с большей активностью симметричных колебаний по сравнению с антисимметричными.

Противоположным примером может служить одиночная диффузная полоса (ширина $\approx 300 \mathrm{~cm}^{-1}$ ) с центром около $2950 \mathrm{~cm}^{-1}$. В случае ориентации $\mathbf{E} \| Z$ для образцов $2 \mathrm{~S}-4 \mathrm{~S}$ для этой полосы величина $\rho>3 / 4$. При $\mathbf{E} \perp Z$ интенсивность полосы $2950 \mathrm{~cm}^{-1}$ для образцов $2 \mathrm{~S}-4 \mathrm{~S}$ становится заметно сильнее, а $\rho$ уменьшается до значений $<3 / 4$ (рис. 5). Полоса $2950 \mathrm{~cm}^{-1}$ относится к колебаниям комплекса гидроксония с кластером воды $\mathrm{H}_{3} \mathrm{O}^{+}\left(\mathrm{H}_{2} \mathrm{O}\right)_{n}$ [37-40]. Характер степени деполяризации полосы $2950 \mathrm{~cm}^{-1}$ в спектрах КР указывает на ориентацию комплекса $\mathrm{H}_{3} \mathrm{O}^{+}\left(\mathrm{H}_{2} \mathrm{O}\right)_{n}$ относительно $c$-каналов апатита и высокую активность антисимметричной моды комплекса. Для ориентаций $\mathbf{E} \| Z$ и $\mathbf{E} \perp Z$ в окрестности $2950 \mathrm{~cm}^{-1}$ в спектрах КР, исходя из анализа значений $\rho$, по преимуществу возбуждаются антисимметричные и симметричные моды комплекса $\mathrm{H}_{3} \mathrm{O}^{+}\left(\mathrm{H}_{2} \mathrm{O}\right)_{n}$ соответственно.

\section{Заключение}

Методами ИК и КР-спектроскопии проведены поляризационные исследования колебательных спектров кластеров $\left(\mathrm{H}_{2} \mathrm{O}\right)_{n}$ в образцах монокристаллического фторапатита разных месторождений. Показано, что особенности взаимодействия внешнего электромагнитного поля и кластеров нестехиометрической воды $\left(\mathrm{H}_{2} \mathrm{O}\right)_{n}$ с группами F...HO, находящимися внутри $c$-каналов апатита, могут быть объяснены в рамках диполь-дипольного приближения. Показано, что это взаимодействие имеет индукционно-резонансный характер, обусловленный переносом колебательного (метод ИК спектроскопии) и электронно-колебательного (метод спектроскопии КР) возбуждения от групп F...НО, которые активно взаимодействуют с внешним полем, к кластерам $\left(\mathrm{H}_{2} \mathrm{O}\right)_{n}$. Из приведенных данных следует, что количественный анализ волы в минералах со структурой типа апатита должен учитывать влияние групп типа F. . .HO на поглощающие и рассеивающие свойства нестехиометрической воды $\left(\mathrm{H}_{2} \mathrm{O}\right)_{n}$, находящейся в микротрещинах минералов.

Полученные результаты могут быть использованы при создании оптических сред, обеспечивающих более эффективное взаимодействие внешнего излучения с рабочими центрами, путем введения в среду подходящих примесей, способных активировать рабочие центры с учетом рассмотренного в работе механизма возбуждения.

Исследования спектров КР проведены с использованием оборудования ресурсного центра Научного парка СПбГУ „Оптические и лазерные методы исследования вещества“. Приношу благодарность Д. Панькину за измерения спектров КР апатитов и обсуждение результатов.

\section{Список литературы}

[1] Sheng Y.M., Gong B., Li W.C., Xia M. // Sci. China. Earth Sci. 2016. V. 59. P. 901-909. doi 10.1007/s11430-016-5281-0

[2] Смирнов С.3., Томас В.Г., Соколова Е.Н., Куприянов И.Н. // Геология и геофизика, 2011. Т. 52. № 5. C. $690-703$.

[3] Штенберг М.В., Игуменцева М.А., Быков В.Н. // ЛИТОСФЕРА, 2010. № 4. C. 152-156

[4] Grundy W.M., Young L.A., Spencer J.R., et al. // Icarus. 2006. V. 184. P. $543-555$.

[5] Staby E., Koch-Muller M., Forster Hans-Jurgen, Wirth R., Rhede D., Schreiber A., Schade U. // Meteor. Planet. Sci. 2016. P. 1-17. doi 10.1111/maps. 12598

[6] Robinson K.L. Water in evolved lunar rocks. PhD dissertation. University of Hawai. 2015.

[7] Warner B.D., Harris A.W., Pravec P. // Icarus. 2009. V. 202. P. 134-146.

[8] Boctor N.Z., Alexander C.M.O'D., Wang J., Hauri E. // Geochim. Cosmochim. Acta. 2003. V. 67. P. 3971-3989.

[9] Wilson E.E., Awonusi A., Morris M.D., Kohn D.H., Tecklenburg M.M.J., Beck L.W. // J. Bone and Mineral Res. 2005. V. 20. P. 625-634.

[10] Rey C., Combes C., Drouet C., Glimcher M.J. // Osteoporosis International. 2009. V. 20. N 6. P. 1013-1021. doi 10.1007/s00198-009-0860-y

[11] Аврунин А.С., Денисов-Никольский Ю.И., Докторов А.А., Кривосенко Ю.С., Самойленко Д.О., Павлычев А.А., Шубняков И.И. // Травм. и ортопед. России. 2015. Т. 3. С. 37.

[12] Soejoko D.S., Sari Y.W., Dewi S.U., Nurizati, Dahlan K., Atmadja D.S. // J. Med. Phys. Biop. 2014. V. 1. N 1. P. 2-7.

[13] Pasteris J.D., Yoder C.H., Wopenka B. // Amer. Mineralogist. 2014. V. 99. N 1. P. 16. doi 10.2138/am.2014.4627

[14] Wilson E.E., Awonusi A., Morris M.D., Kohn D.H., Tecklenburgy M.M.J., Beck L.W. // Biophys. J. 2006. V. 90. P. 3722-3731. doi 10.1529/biophysj.105.070243

[15] Karen L. Wang, Youxue Zhang, Fabian U.N. // Amer. Mineralogist. 2011. V. 96. P. 1392. doi 10.2138/am.2011.3756

[16] Freund F., Knobel R.M. // J. Chem. Soc. Dalton Trans. 1977. V. 11. P. $1136-1140$.

[17] Ceponkus J., Engdahl A., Uvdal P., Nelander B. // Chem. Phys. Lett. 2013. V. 581. P. 1-9.

[18] Lenz A., Ojamale L. // J. Phys. Chem. A. 2006. V. 110. P. $13388-13393$.

[19] Banwell C.N. Fundamentals of Molecular Spectroscopy. McGraw-Hill, 1983. Перевод: Бенуэлл К. Основы молекулярной спектроскопии. М.: Мир, 1985.

[20] McHale J.L. Molecular Spectroscopy. Prentice Hall, 1999. $463 \mathrm{p}$.

[21] Вилков Л.В., Пентин Ю.А. Физические методы исследования в химии. Структурные методы и оптическая спектроскопия. М.: Высшая школа, 1987. 367 с.

[22] Ермолаев В.Л., Бодунов Е.Н., Свешникова Е.Б., Шахвердов T.A. Безызлучательный перенос энергии электронного возбуждения. Л.: Наука, 1977. 238 с.

[23] Ермолаев В.Л., Свешникова Е.Б., Бодунов Е.Н. // УФН. 1996. T. 166. B. 3. C. $279-302$.

[24] Агранович В.М., Галанин М.Д. Перенос энергии возбуждения в конденсированных средах. М.: Наука, 1978. 383 с.

[25] Englman R. Non-Radiative Decay of Ions and Molecules in Solids. Amsterdam: North Holland, 1979. 336 p. 
[26] Корниенко H.Е. Аномальное возрастание ИК поглощения для последовательности дипольной моды $\mathrm{U}_{3}\left(\mathrm{~F}_{2}\right)\left(\mathrm{SiO}_{4}\right)$ монокристаллического и дисперсного кварца. Электронный ресурс. Режим доступа: http://physics.by/e107_files/ /mono/book2007_2pdf/20072st_57.pdf

[27] Золотарев В.М. // Опт. и спектр. 2017. Т. 123. № 5. C. 717-727.

[28] Clark R.N., Swayze G.A., Wise R., Livo K.E., Hoefen T.M., Kokaly R.F., Sutley S.J. USGS Digital Spectral Library splib06a. U.S. Geological Survey. Data Series 231. 2007. Электронный ресурс. Режим доступа: https://crustal.usgs.gov/speclab

[29] Baldridge A.M., Hook S.J., Grove C.I., Rivera G. // Remote Sensing of Environment. 2009. V. 113. P. 711-715. The ASTER Spectral Library Version 2.0. Электронный ресурс. Режим доступа https://speclib.jpl.nasa.gov/

[30] Кубелка П. Спектроскопия отражения. Теория, методы, техника. М.: Мир, 1978.

[31] Auslender M., Hava S. // Handbook of Optical Constants of Solids / Ed. by Palik E.D. San Diego: Acad. Press, 1996. V. 3. P. 155 .

[32] Kristinaityté K., Dagys L., Kausteklis J., Klimavicius V., Doroshenko I., Pogorelov V., Valevičienè N.R., Balevicius V. // J. Molec. Liq. 2017. V. 235. P. 1-6.

[33] Otto K.E., Xue Z., Zielke P., Suhm M.A. // Phys. Chem. Chem. Phys. 2014. V. 16. P. 9849.

[34] Yu H., Cui Q. // J. Chem. Phys. 2007. V. 127. P. 234504.

[35] Abramczyk H., Surmacki J., Brozek-Pluska B., Kordek R. // J. Bioph. Chem. 2011. V. 2. N 2. P. 5154. doi $10.4236 /$ jbpc. 2011.22020

[36] Козлов Н.П., Полянская О.М. // Вестник СамГУ. Естественно-научная серия. 2006. № 3(43). С. 94-99.

[37] Юхневич Г.В. Инфракрасная спектроскопия воды. М.: Наука, 1973. 208 c.

[38] Fournier J.A., Wolke C.T., Johnson C.J. et al. // PNAS. 2014. V. 111. N 51. P. 18132. doi 10.1073/pnas.1420734111

[39] Fournier J.A. et al. // Science. 2014. V. 344. P. 1009-1012.

[40] Shin J.-W. et al. // Science. 2004. V. 304. P. 1137-1140. 